\title{
Ten-year trends in antibiotic usage at a tertiary care hospital in Korea, 2004 to 2013
}

\author{
Bongyoung Kim ${ }^{1,2}$, Hyeonjun Hwang ${ }^{3}$, Jieun $\mathrm{Kim}^{1}$, Myoung-jae Lee ${ }^{4}$, and Hyunjoo Pai ${ }^{1}$
}

\begin{abstract}
${ }^{1}$ Department of Internal Medicine, Hanyang University College of Medicine, Seoul; ${ }^{2}$ Department of Internal Medicine, Eulji University School of Medicine, Daejeon, Korea; ${ }^{3}$ School of Economic Sciences, Washington State University, Pullman, WA, USA; ${ }^{4}$ Department of Economics, Korea University College of Political Science and Economics, Seoul, Korea
\end{abstract}

Received: September 29, 2017 Revised : January 20, 2018 Accepted: March 3, 2018

\section{Correspondence to}

Hyunjoo Pai, M.D.

Department of Internal

Medicine, Hanyang University

College of Medicine, 222-1

Wangsimni-ro, Seongdong-gu,

Seoul 04763, Korea

Tel: $+82-2-2290-8356$

Fax: +82-2-2298-9183

E-mail: paihj@hanyang.ac.kr
Background/Aims: This study was performed to evaluate trends in antibiotic usage at a tertiary care hospital in Korea.

Methods: This study collated antibiotic prescription data and total patient days for inpatients at a tertiary care hospital in Korea between 2004 and 2013. The consumption of each class of antibiotic was converted to defined daily dose (DDD)/1,000 patient-days. We defined 3rd generation cephalosporins, 4th generation cephalosporins, $\beta$-lactam/ $\beta$-lactamase inhibitors, and fluoroquinolones as broad-spectrum antibiotics; carbapenems, tigecycline, glycopeptides, oxazolidinone, and polymyxin were defined as antibiotics against multidrug-resistant (MDR) pathogens. Other antibiotic classes were defined as nonbroad-spectrum antibiotics.

Results: Mean antibiotic consumption was 920.69 DDD/1,000 patient-days. The proportions of broad-spectrum antibiotics, antibiotics against MDR pathogens, and nonbroad-spectrum antibiotics were 41.8\% (384.48/920.69), 3.5\% (32.24/920.69), and $54.7 \%$ (503.97/920.69), respectively. Consumption of broad-spectrum antibiotics (coefficient for time $0.141 ; p=0.049$ ) and antibiotics against MDR pathogens (coefficient for time $0.185 ; p<0.001$ ) showed a significant increasing trend over the study period. Nonbroad-spectrum antibiotic consumption showed a significant decreasing trend over the study period (coefficient for time $-2.601 ; p<0.001$ ).

Conclusions: Over the 10-year period, a stepwise increase in the consumption of broad-spectrum antibiotics and antibiotics against MDR pathogens was observed at a tertiary care hospital in Korea. Conversely, during the same period, nonbroad-spectrum antibiotic consumption showed a significant decreasing trend.

Keywords: Anti-bacterial agents; Hospitals; Korea

\section{INTRODUCTION}

Antimicrobial resistance is currently one of the greatest public health threats and an emerging crisis for humans [1]. Once a pathogen acquires resistance, it erodes the effectiveness of antibiotics, leading to increased mortality, length of hospital stay, and medical costs [2]. Unfortunately, the introduction of new licensed antibi- otics has dwindled since the 1990s due to technical difficulties, regulatory hurdles, and the fact that antibiotics are less-lucrative than other pharmaceuticals [3].

The emergence of antimicrobial-resistant organisms is mainly caused by excessive and inappropriate antibiotic usage [4]. The proportion of inappropriate antibiotic prescription, which induces selective pressure, is approximately $20 \%$ to $50 \%$ worldwide [5]. Therefore, the importance of the antimicrobial stewardship programs 
(ASPs) has been emphasized [5]. Accordingly, the Korean Ministry of Health and Welfare established the Korean National Action Plan on Antimicrobial Resistance in 2016 [6].

The first step to implement proper antimicrobial stewardship policy is identifying the current situation. According to the 'seven core elements' of successful ASPs proposed by the Centers for Disease Control and Prevention, monitoring antibiotic use and resistance patterns is indispensable [7]. In Korea, available data on antibiotic consumption at hospital level are still limited.

This study was performed to evaluate the amount of antibiotic consumption and trends in antibiotic usage at hospital level in Korea. To this end, we obtained and analyzed antibiotic prescription data for inpatients at a tertiary care hospital between 2004 and 2013.

\section{METHODS}

\section{Study design and setting}

Hanyang University Seoul Hospital is a 758-bed, university-affiliated tertiary care hospital located in Seoul, South Korea. It has two intensive care units (ICUs), including a medical ICU (14 beds) and a surgical ICU (15 beds). Some changes in ASPs were observed in this hospital during the study period. In 2007, the Health Insurance Review \& Assessment Service initiated an evaluation of surgical prophylactic antibiotics and implemented controls on prophylactic antibiotics used in surgery. Furthermore, the preauthorization-of-antibiotic-use program was newly implemented in 2008, applicable to the following antibiotics: carbapenems, glycopeptides, oxazolidinone, and polymyxin. Preauthorization for tigecycline was introduced in 2009. Other ASPs, including education for proper antibiotic use and feedback to the prescriber after drug use evaluation were largely unchanged during the study period although these practices were reinforced in 2008. The major events in infection control measures against multidrug-resistant (MDR) organisms during the study period were: (1) during 2011, the isolation ward was operated but closed in the following year; (2) the medical ICU participated in a multicenter study for MDR Acinetobacter baumannii control between 2011 and 2012 and, as part of this, active surveillance culture for MDR A. baumannii was performed and terminal environmental cleaning was reinforced; and (3) alcohol gel for hand hygiene was introduced at every patient's bedside in 2010 .

Data on total antibiotic prescriptions, patient-days, and antimicrobial sensitivity tests among inpatients between 2004 and 2012 from Hanyang University Seoul Hospital were collected for this study.

\section{Definitions}

\section{Antibiotics}

In this paper, we defined antibiotics as medication classified as class Jor according to the Anatomical Therapeutic Chemical (ATC) classification system, which does not include antifungal agents or anti-tuberculosis agents. Systemic agents used via oral or parenteral administration routes are included while topical agents are excluded. We converted each class antibiotics amount to defined daily dose (DDD) by using the ATC classification system by the World Health Organization [8], and then standardized for 1,000 patient-days.

We classified antibiotic agents into 19 classes: 1st generation cephalosporins (1st CEPs), 2nd generation cephalosporins (2nd CEPs), 3rd generation cephalosporins (3rd CEPs), 4th generation cephalosporins (4th CEPs), aminoglycosides (AGs), $\beta$-lactam/ $\beta$-lactamase inhibitors (BL/BLIs), carbapenems, fluoroquinolones (FQs), glycopeptides, lincosamides, macrolides, metronidazole, monobactam, oxazolidinone, penicillins, polymyxin, tetracyclines, tigecycline, and trimethoprim/sulfamethoxazole (SXT). Other antibiotics, such as amphenicol, fosfomycin, and streptogramin were excluded because they are rarely used.

We defined 3rd CEPs, 4 th CEPs, BL/BLIs, and FQs as broad-spectrum antibiotics. Carbapenems, tigecycline, glycopeptides, oxazolidinone, and polymyxin were defined as antibiotics against MDR pathogens. The remaining antibiotic classes were defined as nonbroad-spectrum antibiotics.

Bacterial pathogens and antimicrobial resistance

We analyzed antimicrobial sensitivity tests for bacterial pathogens isolated from blood. Escherichia coli, Klebsiella pneumoniae, and Staphylococcus aureus were selected for analysis because they are commonly associated with antimicrobial resistance and a relatively high isolation 


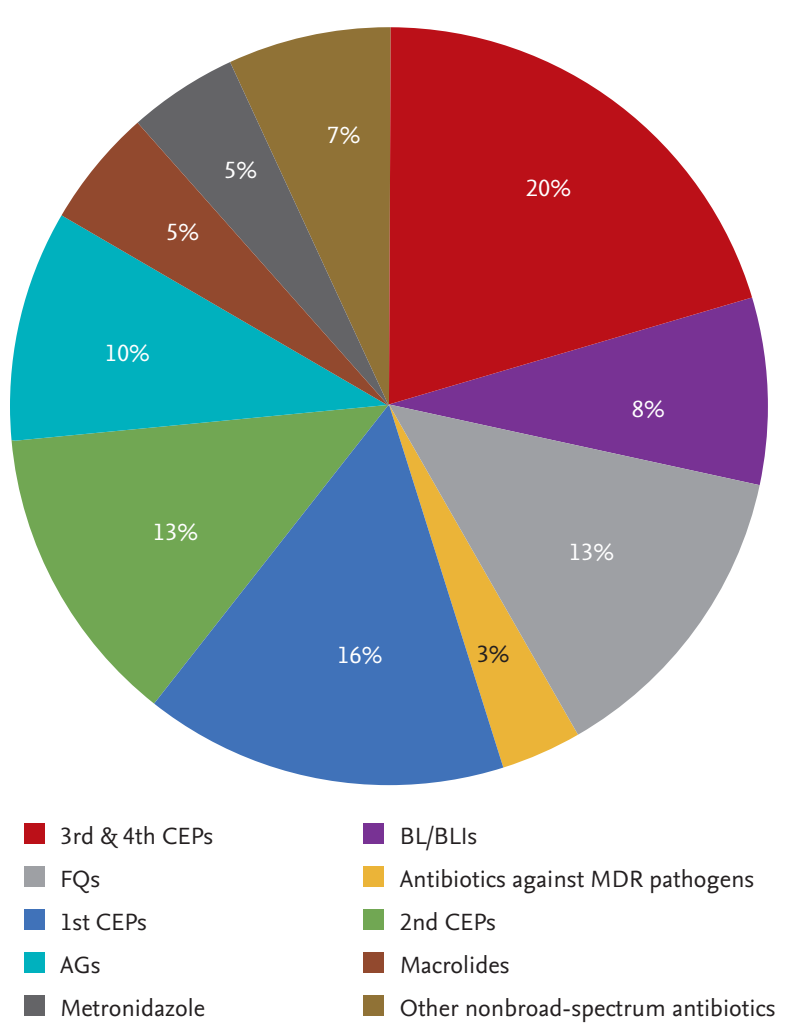

Figure 1. The proportion of consumption of antimicrobial agents for systemic use by class at a tertiary care hospital in Korea, 2004 to 2013. CEP, cephalosporin; BL, $\beta$-lactam; BLI, $\beta$-lactamase inhibitor; FQ, fluoroquinolone; MDR, multidrug-resistant; AG, aminoglycoside.

rate. The first isolate for each pathogen obtained from a single patient during a hospitalization period was included and duplicated isolates were excluded. All isolates were identified with MicroScan Walkaway (Beckman Coulter, Brea, CA, USA) and Vitek automated bacterial identification system 2 (bioMèrieux, Marcy-I'Etoile, France). The breakpoints of each compound were defined in reference to the Clinical and Laboratory Standards Institute (CLSI) [9], and R (resistance) or I (intermediate) were defined as resistance.

\section{Statistical analysis}

A linear regression model was used to assess the trend of antibiotic consumption and antimicrobial resistance rate over time. The coefficient for time and $p$ value for the trend of antibiotic consumption were extracted using measures on a monthly basis while those for the trend of antimicrobial resistance rate were extracted using measures on a yearly basis because isolates were not sufficient for statistical power. Statistical significance was defined as $p<0.05$. All analyses were performed using SPSS version 24.0 for Windows (IBM Co., Armonk, NY, USA).

\section{Ethical statement}

The study protocol was approved by the Institutional Review Boards of Eulji University Hospital (IRB number: 2017-06-021), and the requirement for written informed consent from patients was waived.

\section{RESULTS}

\section{Ten-year trend of antibiotic consumption}

Over the 10-year study period, the mean antibiotic consumption was $920.69 \mathrm{DDD} / 1,000$ patient-days. The proportion of broad-spectrum antibiotics, antibiotics against MDR pathogens and nonbroad-spectrum antibiotics were $41.8 \%$ (384.48/920.69), 3.5\% (32.24/920.69), and $54.7 \%$ (503.97/920.69), respectively. The most commonly prescribed antibiotic class was 3rd CEPs (18.8\%, 173.26/920.69), followed by 1st CEPs (15.5\%, 143.10/920.69), FQs (13.4\%, 123.15/920.69), 2nd CEPs (12.7\%, 116.94/ 920.69), AGs (9.8\%, 90.57/920.69), and BL/BLIs (8.0\%, 74.11/920.69) (Fig. 1).

Table 1 shows the overall annual consumption of antimicrobial agents for systemic use. The overall annual antibiotic consumption ranged from 815.10 (in 2010) to 1,047.96 (in 2006) and showed a significant decreasing trend (coefficient for time -2.274; $p<0.001$ ). A significant increasing trend in the use of broad-spectrum antibiotics (coefficient for time $0.141 ; p=0.049$ ) and antibiotics against MDR pathogens (coefficient for time 0.185 ; $p<0.001$ ) was observed over the study period. Conversely, nonbroad-spectrum antibiotics showed a significant decreasing trend between 2004 and 2013 (coefficient for time $-2.601 ; p<0.001$ ) (Fig. 2A).

Looking at the trends by classes, a significant increasing trend was observed for 4 th CEPs (coefficient for time 0.223; $p<0.001$ ) and BL/BLIs (coefficient for time $0.261 ; p<0.001$ ) while a significant decreasing trend was observed for 3 rd CEPs (coefficient for time -0.295 ; $p<$ $0.001)$. The consumption of FQs remained stable throughout the study period (coefficient for time $-0.048 ; p=0.199$ ) (Fig. 2B). 


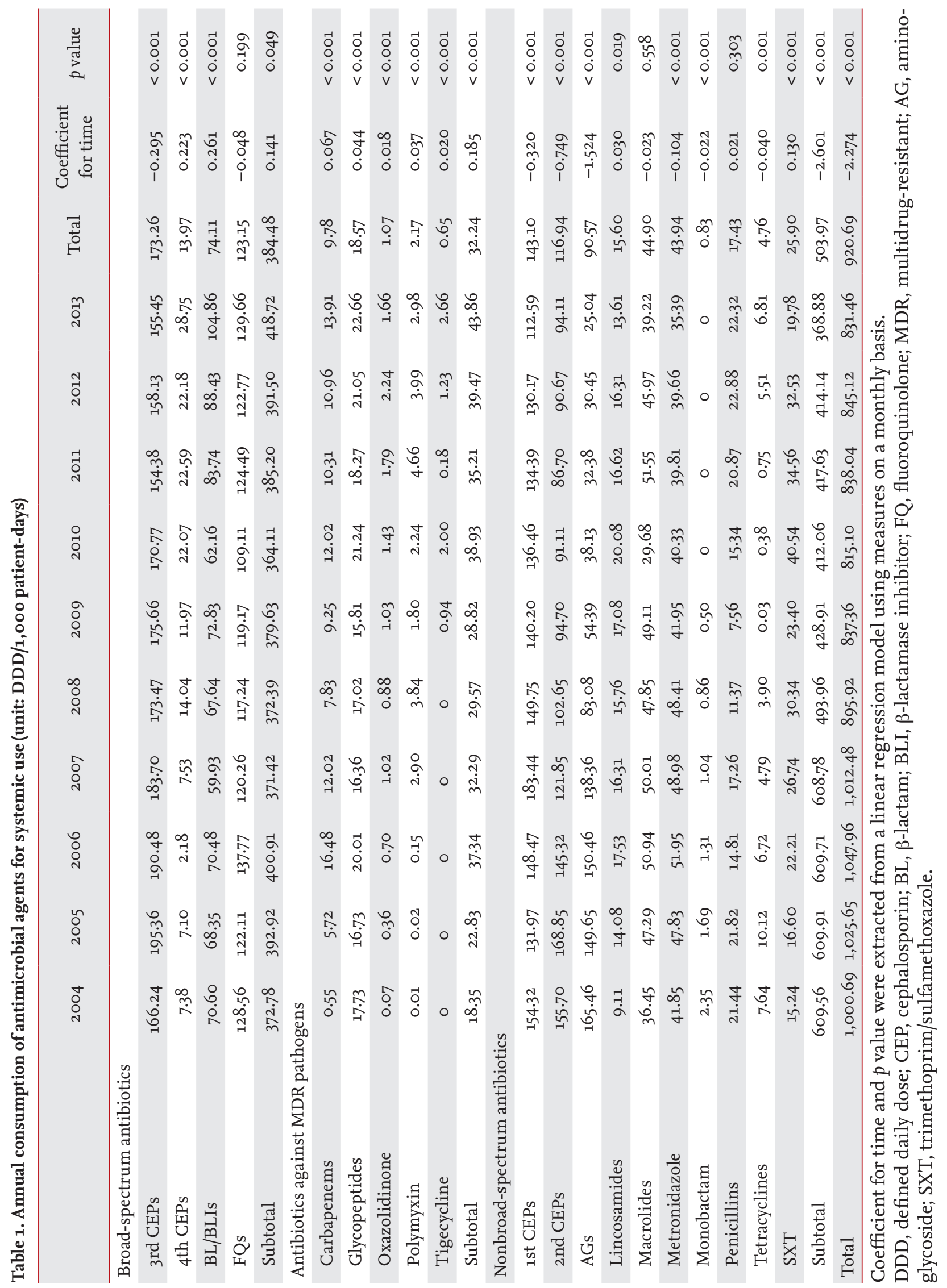



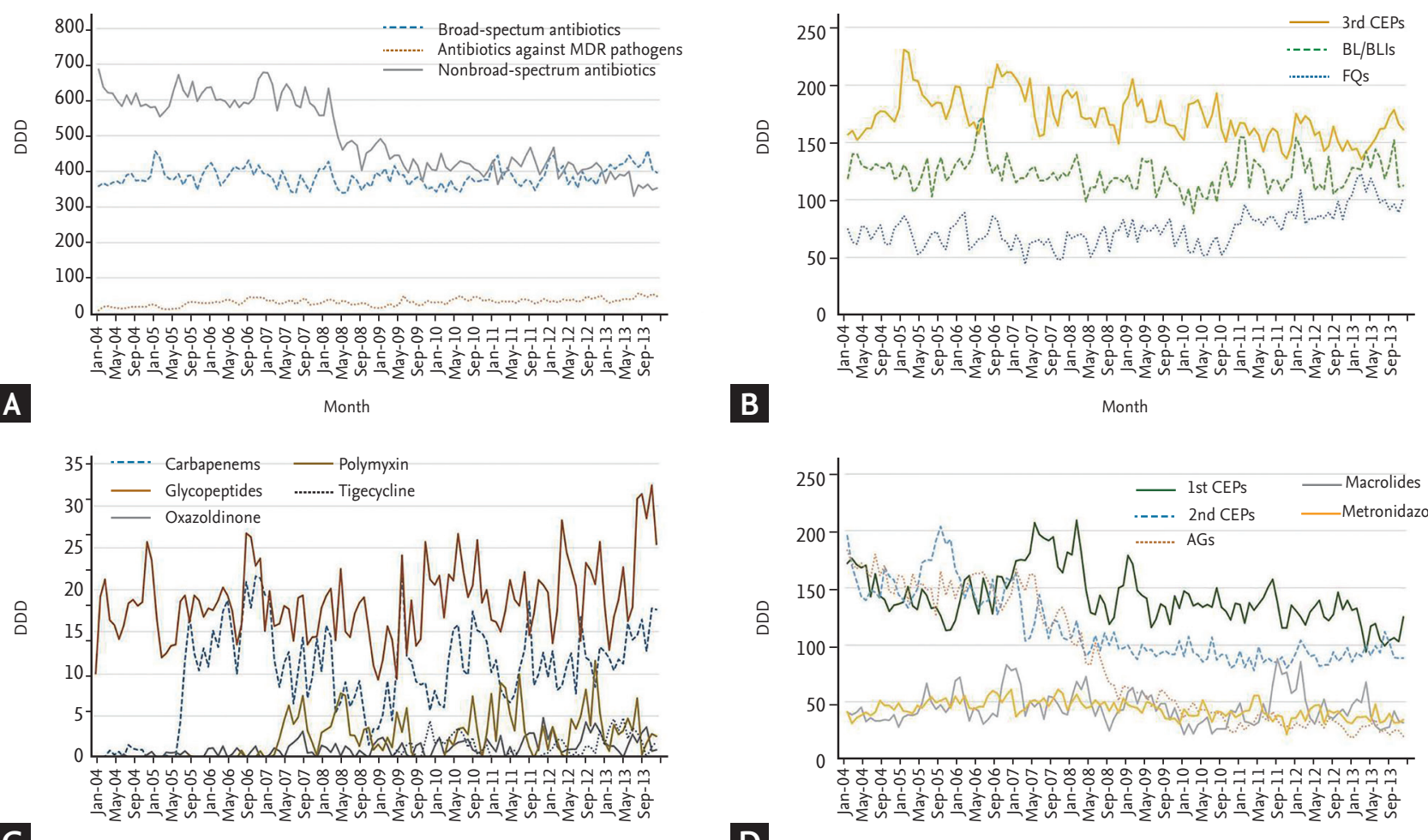

C

Month

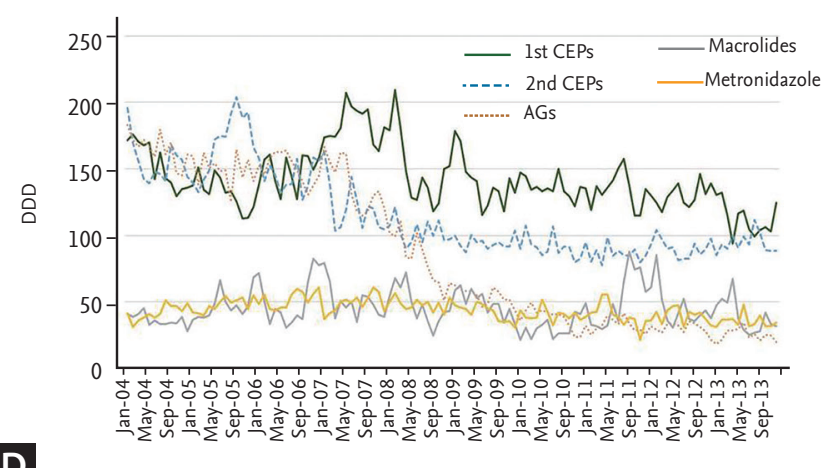

D

Month

Figure 2. Ten-year trends of consumption of antimicrobial agents for systemic use at a tertiary care hospital in Korea, 2004 to 2013 (unit: defined daily dose [DDD]/1,00o patient-days). (A) Overall. (B) Broad-spectrum antibiotics. (C) Antibiotics against multidrug-resistant pathogens. (D) Nonbroad-spectrum antibiotics. MDR, multidrug-resistant; CEP, cephalosporin; BL, $\beta$-lactam; BLI, $\beta$-lactamase inhibitor; FQ, fluoroquinolone; AG, aminoglycoside.

Significant increasing trends were observed throughout the study period for consumption of all classes of antibiotics against MDR pathogens: carbapenems (coefficient for time $0.067 ; p<0.001$ ), glycopeptides (coefficient for time $0.044 ; p<0.001$ ), oxazolidinone (coefficient for time $0.018 ; p<0.001$ ), polymyxin (coefficient for time $0.037 ; p<0.001$ ), and tigecycline (coefficient for time $0.020 ; p<0.001$ ) (Fig. 2 C).

For most of the nonbroad-spectrum antibiotics, consumption showed significant decreasing trends: 1st CEPs (coefficient for time $-0.320 ; p<0.001$ ), 2nd CEPs (coefficient for time $-0.749 ; p<0.001$ ), AGs (coefficient for time -1.524; $p<0.001$ ), metronidazole (coefficient for time $-0.104 ; p<0.001$ ), monobactam (coefficient for time $-0.022 ; p<0.001$ ), and tetracycline (coefficient for time $-0.040 ; p=0.001$ ). However, lincosamide (coefficient for time $0.030 ; p=0.019$ ) and SXT (coefficient for time $0.130 ; p<0.001$ ) consumption showed a significant increasing trend. The consumption of macrolides and penicillins remained stable throughout the study period (Fig. 2D).

\section{Consumption of antibiotics stratified by ward and department}

Table 2 shows consumption of antimicrobial agents for systemic use by ward and department. Mean antibiotic consumption was 1.7 times higher among patients in the ICUs than among patients in the general wards (GWs) (1,568.16 DDD vs. 897.39 DDD). Furthermore, in the ICUs, mean consumption of broad-spectrum antibiotics was 2.1 times higher (769.84 DDD vs. 370.61 DDD) and mean consumption of antibiotics against MDR pathogens was 9.2 times higher (231.00 DDD vs. 25.08 DDD) than in the GWs. The mean nonbroad-spectrum antibiotic consumption was comparable between patients in ICUs and GWs (567.32 DDD vs. 501.70 DDD). In the ICUs, a significant increasing trend (coefficient for time $0.655 ; p=0.011$ ) was found for consumption of anti- 
Table 2. Consumption of antimicrobial agents for systemic use by wards and departments (unit: DDD/1,000 patient-days)

\begin{tabular}{|c|c|c|c|c|}
\hline & \multicolumn{2}{|c|}{ Wards } & \multicolumn{2}{|c|}{ Departments } \\
\hline & Intensive care units & General wards & $\begin{array}{c}\text { Surgical } \\
\text { departments }\end{array}$ & $\begin{array}{c}\text { Medical } \\
\text { departments }\end{array}$ \\
\hline \multicolumn{5}{|c|}{ Broad-spectrum antibiotics } \\
\hline 3rd CEPs & 336.10 & 167.40 & 253.04 & 107.12 \\
\hline 4th CEPs & 50.76 & 12.65 & 5.97 & 20.61 \\
\hline BL/BLIs & 172.71 & 70.56 & 111.47 & 43.14 \\
\hline FQs & 210.26 & 120.01 & 89.73 & 150.84 \\
\hline Subtotal & 769.84 & 370.61 & 460.21 & 321.71 \\
\hline \multicolumn{5}{|c|}{ Antibiotics against MDR pathogens } \\
\hline Carbapenems & 82.11 & $7 \cdot 17$ & 5.27 & 13.51 \\
\hline Glycopeptides & 99.00 & 15.67 & 23.83 & 14.21 \\
\hline Oxazolidinone & 8.80 & 0.79 & 0.92 & 1.20 \\
\hline Polymyxin & 32.88 & 1.06 & 1.28 & 2.90 \\
\hline Tigecycline & 8.21 & 0.38 & 0.45 & 0.82 \\
\hline Subtotal & 231.00 & 25.08 & 31.75 & 32.64 \\
\hline \multicolumn{5}{|c|}{ Nonbroad-spectrum antibiotics } \\
\hline 1st CEPs & $45 \cdot 46$ & 146.62 & 255.23 & 50.16 \\
\hline 2nd CEPs & $53 \cdot 53$ & 119.22 & 210.54 & $39 \cdot 35$ \\
\hline AGs & 219.88 & 85.92 & $144 \cdot 44$ & $45 \cdot 93$ \\
\hline Lincosamides & 82.90 & 13.21 & 4.19 & 25.05 \\
\hline Macrolides & 19.14 & 45.83 & $14 \cdot 57$ & 70.04 \\
\hline Metronidazole & 71.39 & 42.95 & 48.19 & 40.42 \\
\hline Monobactam & $4 \cdot 47$ & 0.70 & 1.09 & 0.61 \\
\hline Penicillins & 22.96 & 17.23 & 15.79 & 18.78 \\
\hline Tetracyclines & 9.69 & 4.58 & 3.07 & 6.16 \\
\hline SXT & 38.71 & $25 \cdot 44$ & 16.48 & 33.71 \\
\hline Subtotal & 567.32 & 501.70 & 713.60 & 330.21 \\
\hline Total & $1,568.16$ & 897.39 & $1,205.57$ & 684.56 \\
\hline
\end{tabular}

DDD, defined daily dose; CEP, cephalosporin; BL, $\beta$-lactam; BLI, $\beta$-lactamase inhibitor; FQ, fluoroquinolone; MDR, multidrug-resistant; AG, aminoglycoside; SXT, trimethoprim/sulfamethoxazole.

biotics against MDR pathogens while nonbroad-spectrum antibiotics showed a significant decreasing trend (coefficient for time -2.629; $p<0.001$ ) throughout the study period. The consumption of broad-spectrum antibiotics remained stable (coefficient for time -0.024; $p$ $=0.941$ ). Similarly, in the GWs, a significant increasing trend was observed for the consumption of antibiotics against MDR pathogens (coefficient for time 0.156; $p<$ o.001) while a significant decreasing trend was observed for nonbroad-spectrum antibiotics (coefficient for time $-2.580 ; p<0.001$ ). The consumption of broad-spectrum antibiotics remained stable between 2004 and 2013 (coefficient for time 0.121; $p=0.087$ ) (Supplementary Tables 1 and 2).

Mean antibiotic consumption among patients in surgical departments was 1.8 times higher than among patients in medical departments (1,205.57 DDD vs. 684.56 DDD). Compared to patients in medical departments, mean consumption of broad-spectrum antibiotics among patients in surgical departments was approximately 1.4 times higher (460.21 DDD vs. 321.71 DDD) and mean consumption of nonbroad-spectrum antibi- 
otics (731.6o DDD vs. 330.21 DDD) was 2.2 times higher. There was a slightly higher consumption of antibiotics against MDR among patients in medical departments compared to patients in surgical departments (31.75 DDD vs. 32.64 DDD). In surgical departments, antibiotics against MDR pathogens had a significant increasing trend (coefficient for time 0.149; $p<0.001$ ) while broad-spectrum antibiotics (coefficient for time -0.939 ; $p$ $<0.001$ ) and nonbroad-spectrum antibiotics (coefficient for time -4.163; $p<0.001$ ) showed a significant decreasing trends over the study period. Consumption of broad-spectrum antibiotics (coefficient for time 1.096; $p$ $<0.001$ ) and antibiotics against MDR pathogens (coefficient for time $0.216 ; p<0.001$ ) showed a significant increasing trend while nonbroad-spectrum antibiotic consumption (coefficient for time $-1.180 ; p<0.001$ ) showed a significant decreasing trend over the study period (Supplementary Tables 3 and 4).

\section{Trends in antimicrobial resistance of bacterial pathogens}

Table 3 shows the 10-year trend in antimicrobial resistance for E. coli, K. pneumoniae, and S. aureus. Looking at the gram-negative pathogens, the E. coli and K. pneumoniae resistance rates to ciprofloxacin were $31.8 \%$ and $37.5 \%$, respectively, in 2013 . The $K$. pneumoniae resistance rate to ciprofloxacin increased significantly over the study period (coefficient for time 3.693; $p=0.002$ ). The $E$. coli and $K$. pneumoniae resistance rates to SXT were $34.1 \%$ and $29.2 \%$, respectively, in 2013 . The resistance rate of K. pneumoniae to SXT showed a significant increasing trend over the study period (coefficient for time 2.562; $p=0.022$ ). The production rates of extended-spectrum $\beta$-lactamase (ESBL) in E. coli and K. pneumoniae were $22.7 \%$ and $33.3 \%$, respectively, in 2013 , and had significantly increased (coefficient for time $2.427, p$ $=0.005$, E. coli; coefficient for time 3.727, $p=0.003, \mathrm{~K}$. pneumoniae).

The resistance rate of $S$. aureus, the representative gram-positive pathogen, to clindamycin, oxacillin, and SXT was 47.6\%, 50.0\%, and o\%, respectively, in 2013 . The resistance rate to SXT significantly decreased from 2004 to 2013 (coefficient for time $-2.140 ; p=0.004$ ).

\section{DISCUSSION}

The results from the present study reflect the recent status of antibiotic usage at hospital level in Korea and can be used for implementation of antimicrobial stewardship policies. Few studies analyzing antibiotic usage trend at hospital level have been performed in Korea. Jun et al. [10] reported that average antibiotic consumption among patients at a tertiary care hospital over 18 years of age was $644.6 \mathrm{DDD} / 1,000$ patient-days between 2001 and 2012. Song et al. [11] reported that annual antibiotic consumption among all patients ranged from approximately 750 to $850 \mathrm{DDD} / 1,000$ patient-days at a tertiary hospital between 2000 and 2006.

There was a significant decreasing trend in consumption of nonbroad-spectrum antibiotic throughout the study period. Conversely, the consumption of broadspectrum antibiotics and antibiotics against MDR pathogens increased. This trend was observed both at hospital-level [9,10] and national-level [12,13] analysis in Korea. A comparable trend of antibiotic usage has been observed in other countries. A study conducted in an Italian tertiary care hospital, with a comparable number of beds (840 beds), found significant increasing trends in consumption of BL/BLIs, carbapenems and vancomycin [14]. In acute care hospitals in the UK, use of carbapenems and piperacillin/tazobactam increased by 60.4\% and 94.8\%, respectively [15]. Similarly, data from European Surveillance of Antibiotic Consumption (ESAC) projects, which represents European countries, show that consumption of broad-spectrum penicillins, BL/BLI, carbapenems, and polymyxin usage increased significantly in 2013 compared with usage in 2009 [16].

We suggest that these trends reflect the current problem of increasing antimicrobial-resistant pathogens. While we did not analyze pathogens isolated from other sites, we identified a significant increase in the antimicrobial resistance rate to broad-spectrum antibiotics of bloodstream pathogens isolated at the study hospital. Consistent with our findings, the Korean Antimicrobial Resistance Monitoring System (KARMS), comprising a total of 35 secondary and tertiary care participating hospitals, reported that the antimicrobial resistance rate of E. coli and K. pneumoniae to broad-spectrum antibiotics has been increasing [17]. According to the KARMS, the E. coli and K. pneumoniae resistance 


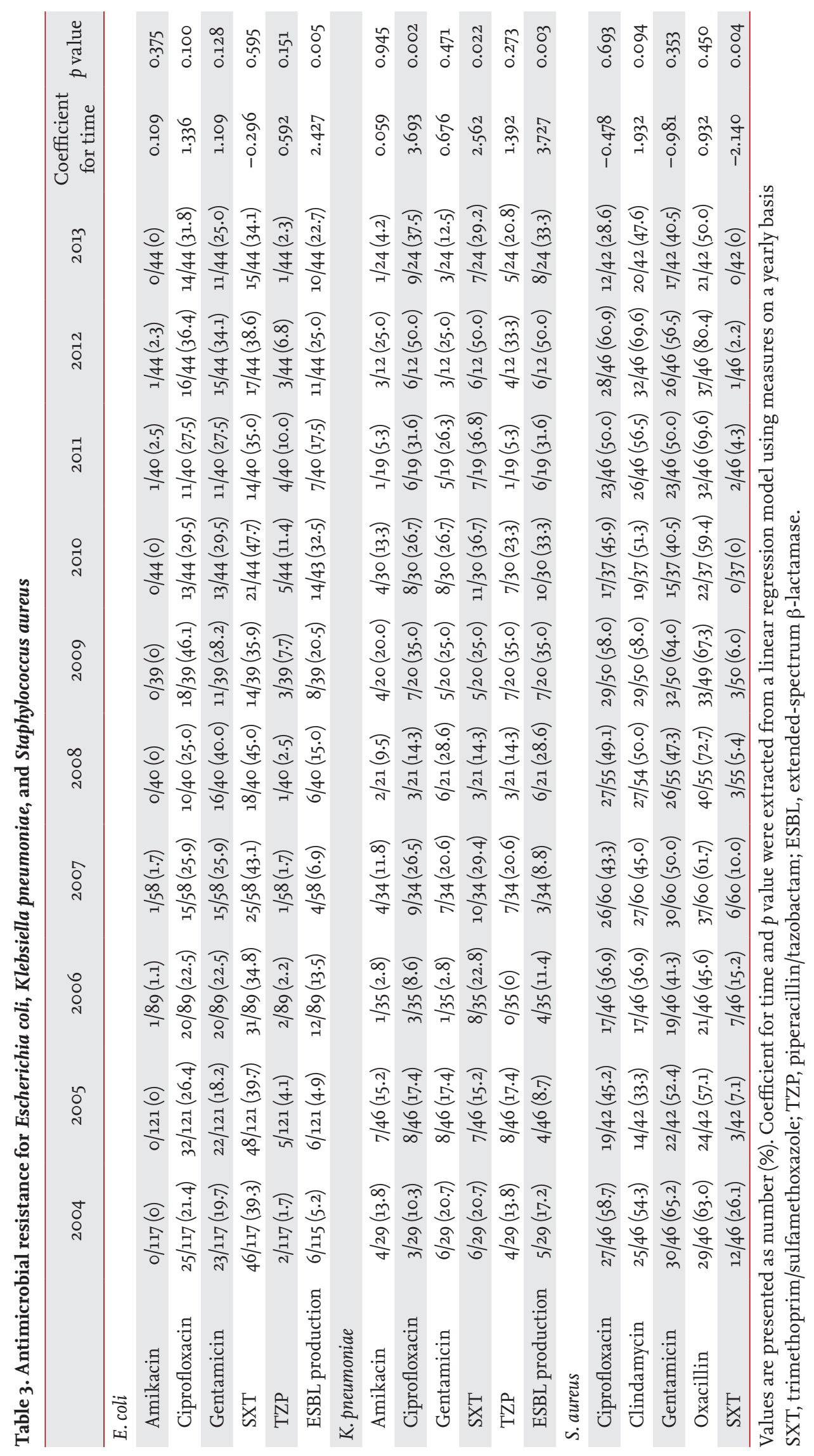


rates to FQs were $30 \%$ in 2004 , increasing to $42 \%$ and $34 \%$, respectively, in 2013 [17]. Similarly, the E. coli and K. pneumoniae resistance rate to cefotaxime were $10 \%$ and $30 \%$, respectively, in 2004 , increasing to $29 \%$ and $40 \%$, respectively, in 2013 [17]. The issue of increasing antimicrobial resistance of gram-negative Enterobacteriaceae is not limited to Korea. A multi-national study in Asian-Pacific countries found that the rate of community-acquired ESBL-producing E. coli and K. pneumonia increased from $6.3 \%$ and $9.4 \%$, respectively, in 2002 to $14.6 \%$ and $26.2 \%$, respectively, in 2013 [18]. This trend resulted in increasing consumption of antibiotics against MDR pathogens, including carbapenems. KARMS reported that the carbapenem susceptibility rates of E. coli and $\mathrm{K}$. pneumoniae decreased from $100 \%$ and $99.3 \%$ in 2011 to $99 \%$ and $97 \%$ in 2015 , respectively [17]. Considering that carbapenems are one of the most reliable last-resort treatment for gram-negative pathogens, this finding is troubling. The high prevalence of resistant gram-positive pathogen is also noticeable. In 2013, we found that half of $S$. aureus isolates from blood were methicillin-resistant $S$. aureus (MRSA). This finding was consistent with results of the KARMS study: the proportion of MRSA between 2013 and 2015 was $66 \%$ to $72 \%$ [17]. Regarding MRSA, the proportion of community-acquired isolates increased from $5.9 \%$ in 2005 to $13.3 \%$ in 2014 [19].

Guidelines for community-based infection may have affected the antibiotic prescription trends of the present study. The Infectious Disease Society of America and the American Thoracic Society introduced the concept of healthcare-associated pneumonia in 2005 and empirical coverage of MDR pathogens, such as MRSA and Pseudomonas aeruginosa, has been promoted since then [20]. Accordingly, the 2007 updated guidelines for community-acquired pneumonia recommended double anti-pseudomonal antibiotic coverage for cases in which Pseudomonas is a consideration; an example is piperacillin/tazobactam plus respiratory FQ combination therapy [21]. The Korean guidelines for community-acquired pneumonia treatment released in 2009 recommend that Pseudomonas infection should be suspected in patients with structural lung diseases such as bronchiectasis, exacerbation of recurrent chronic obstructive lung disease, systemic antibiotic use within the preceding 3 months, and alcoholism [22]. Conse- quently, there has been increased concern among physicians regarding MDR pathogens, driving excessive broad-spectrum antibiotic consumption [23].

We found that patients in ICUs consumed substantially more antibiotics, particularly antibiotics against MDR pathogens, compared with patients in GWs. For patients in ICUs, higher rates of MDR pathogens justify the use of regimens combining different broad-spectrum antibiotics, even when the presumed infection probability is low, because inappropriate empirical therapy may lead to poor prognosis [24]. Furthermore, there has been a significant increase in antimicrobial resistance for major pathogens isolated from ICUs in Korean hospitals [25]. However, study results indicate that $30 \%$ to $60 \%$ of antibiotics used in ICUs are inappropriate and therefore appropriate intervention measures are necessary to prevent further emergence of MDR pathogens, which are highly correlated with selective pressure [24].

ASPs are one of the most demanding strategies to reduce unnecessary and improper antibiotic usage [26]. In the current study, the antibiotic consumption trend may have been affected by ASPs as well. The preauthorization-of-antibiotic-use program was newly implemented in the study hospital in 2008, and other ASPs, such as education for proper antibiotic use and feedback to prescribers after drug use evaluation, were reinforced at the same time. As a result, overall antibiotic consumption began to decrease from this point. However, a rebound in the total antibiotic consumption occurred from 2011. The phenomenon can be explained by lack of manpower. At the beginning of 2011, one infectious diseases specialist resigned from the study hospital and ASPs were operated by one person. As indicated above, the most important requirements for appropriate operation of ASPs in Korea are reinforcement of manpower capable of performing ASPs.

There are potential limitations to the present study. First, the data of our study were derived from records at a tertiary care hospital and many of the patients potentially had underlying illnesses. Furthermore, we did not analyze outpatient data, which account for approximately $80.9 \%$ of total antibiotic consumption [13]. Therefore, the results of the present study may not be applicable to the general population. Secondly, antibiotic consumption was measured by DDD instead of 
days of therapy (DOT). According to a recent guideline for antibiotic stewardship programs, DOT is preferred to DDD as a measure of antibiotic consumption [27]. However, we could not use DOT because only the total amount of antibiotic consumption per patient was available. As one of the known shortcomings of DDD, antibiotic consumption may be underestimated among pediatric patients or patients with decreased renal function. Thirdly, analysis of the antimicrobial resistance rate was limited to three major pathogens isolated from blood. There are other pathogens that reflect the overall status of antimicrobial resistance in a hospital, such as P. aeruginosa, A. baumannii, and Enterococcus faecium. As there were insufficient isolates to analyze trends of antimicrobial resistance rate over time, we were only able to include E. coli, K. pneumoniae, and S. aureus.

Despite these limitations, the data derived from this study are likely to be a reasonable indicator of trends of systemic antibiotic usage at hospital-level. In conclusion, over the 10-year study period, a stepwise increase in the consumption of broad-spectrum antibiotics and antibiotics against MDR pathogens was observed at a tertiary care hospital in Korea. Conversely, over the same period, a significant decreasing trend in the consumption of nonbroad-spectrum antibiotics was observed.

\section{KEY MESSAGE}

1. The proportion of broad-spectrum antibiotics, antibiotics against multidrug-resistant pathogens, and nonbroad-spectrum antibiotics were $41.8 \%, 3.5 \%$, and $54.7 \%$, respectively.

2. Broad-spectrum antibiotics and antibiotics against multidrug-resistant pathogens showed an increasing trend while nonbroad-spectrum antibiotics showed a decreasing trend over the 10-year period.

3. Mean antibiotic consumption was 1.7 times higher among patients in the intensive care units than among patients in the general wards. Notably, patients in intensive care units consumed 9.2 times more antibiotics against multidrug-resistant pathogens compared with patients in general wards.

\section{Conflict of interest}

No potential conflict of interest relevant to this article was reported.

\section{Acknowledgments}

This research was supported by EMBRI Grants 2017 (2017EMBRIDJooo3) from the Eulji University.

\section{REFERENCES}

1. Spellberg B, Guidos R, Gilbert D, et al. The epidemic of antibiotic-resistant infections: a call to action for the medical community from the Infectious Diseases Society of America. Clin Infect Dis 2008;46:155-164.

2. Kollef MH. Broad-spectrum antimicrobials and the treatment of serious bacterial infections: getting it right up front. Clin Infect Dis 2008;47 Suppl 1:S3-S13.

3. Livermore DM. Current epidemiology and growing resistance of gram-negative pathogens. Korean J Intern Med 2012;27:128-142.

4. Angebault C, Andremont A. Antimicrobial agent exposure and the emergence and spread of resistant microorganisms: issues associated with study design. Eur J Clin Microbiol Infect Dis 2013;32:581-595.

5. Dellit TH, Owens R C, McGowan JE Jr, et al. Infectious Diseases Society of America and the Society for Healthcare Epidemiology of America guidelines for developing an institutional program to enhance antimicrobial stewardship. Clin Infect Dis 2007;44:159-177.

6. Ryu S. The new Korean action plan for containment of antimicrobial resistance. J Glob Antimicrob Resist 2017;8:7073 .

7. The Centers for Disease Control and Prevention (CDC). The core elements of hospital antibiotic stewardship programs [Internet]. Atlanta (GA): CDC, 2018 [cited 2018 May 14]. Available from: https://www.cdc.gov/antibiotic-use/ healthcare/pdfs/core-elements.pdf.

8. World Health Organization (WHO) Collaborating Centre for Drug Statistics Methodology. Definition and general considerations [Internet]. Oslo (NO): WHO Collaborating Centre for Drug Statistics Methodology, 2018 [cited 2018 May 14]. Available from: http://www.whocc.no/ddd/ definition_and_general_considera.

9. Clinical and Laboratory Standards Institute (CLSI). M1oo-S15 Performance Standards for Antimicrobial Susceptibility 
Testing: Fifteenth Informational Supplement. Wayne (PA): CLSI, c2005.

10. Jun KI, Koo HL, Kim MK, et al. Trends in antibiotic use in a single university hospital. Korean J Nosocomial Infect Control 2013;18:44-50.

11. Song YA, Jung SI, Chang MO, et al. Relationship between antibiotic use and antibiotic resistance in major nosocomial pathogens at a university hospital. Chonnam Med J 2008;44:137-143.

12. Park J, Han E, Lee SO, Kim DS. Antibiotic use in South Korea from 2007 to 2014: a health insurance databasegenerated time series analysis. PLoS One 2017;12:e0177435.

13. Yoon YK, Park GC, An H, Chun BC, Sohn JW, Kim MJ. Trends of antibiotic consumption in Korea according to national reimbursement data (2008-2012): a population-based epidemiologic study. Medicine (Baltimore) 2015;94:e2100.

14. Mascarello M, Simonetti O, Knezevich A, et al. Correlation between antibiotic consumption and resistance of bloodstream bacteria in a University Hospital in North Eastern Italy, 2008-2014. Infection 2017;45:459-467.

15. Cooke J, Stephens P, Ashiru-Oredope D, et al. Longitudinal trends and cross-sectional analysis of English national hospital antibacterial use over 5 years (2008-13): working towards hospital prescribing quality measures. J Antimicrob Chemother 2015;70:279-285.

16. European Surveillance of Antibiotic Consumption. Summary of the latest data on antibiotic consumption in the European Union 2014 [Internet]. Stockholm (SE): European Surveillance of Antibiotic Consumption, 2014 [cited 2018 May 15]. Available from: https://ecdc.europa.eu/ sites/portal/files/documents/antibiotics-consumption-EUdata-2014.pdf.

17. Kim D, Ahn JY, Lee CH, et al. Increasing resistance to extended-spectrum cephalosporins, fluoroquinolone, and carbapenem in gram-negative bacilli and the emergence of carbapenem non-susceptibility in Klebsiella pneumoniae: analysis of Korean antimicrobial resistance monitoring System (KARMS) data from 2013 to 2015. Ann Lab Med 2017;37:231-239.

18. Chang YT, Coombs G, Ling T, et al. Epidemiology and trends in the antibiotic susceptibilities of Gram-negative bacilli isolated from patients with intra-abdominal infections in the Asia-Pacific region, 2010-2013. Int J Antimicrob Agents 2017;49:734-739.

19. Moon HW, Kim HJ, Hur M, Yun YM. Antimicrobial susceptibility profiles of Staphylococcus aureus isolates classified according to their origin in a tertiary hospital in Korea. Am J Infect Control 2014;42:1340-1342.

20. American Thoracic Society; Infectious Diseases Society of America. Guidelines for the management of adults with hospital-acquired, ventilator-associated, and healthcareassociated pneumonia. Am J Respir Crit Care Med 2005; 171:388-416.

21. Mandell LA, Wunderink RG, Anzueto A, et al. Infectious Diseases Society of America/American Thoracic Society consensus guidelines on the management of communityacquired pneumonia in adults. Clin Infect Dis 2007;44 Suppl 2:S27-S72.

22. Song JH, Jung KS, Kang MW, et al. Treatment guidelines for community-acquired pneumonia in Korea: an evidencebased approach to appropriate antimicrobial therapy. Tuberc Respir Dis 2009;67:281-302.

23. Wunderink RG. Community-acquired pneumonia versus healthcare-associated pneumonia. The returning pendulum. Am J Respir Crit Care Med 2013;188:896-898.

24. Luyt CE, Brechot N, Trouillet JL, Chastre J. Antibiotic stewardship in the intensive care unit. Crit Care 2014;18:480.

25. Choi JY, Kwak YG, Yoo H, et al. Trends in the distribution and antimicrobial susceptibility of causative pathogens of device-associated infection in Korean intensive care units from 2006 to 2013: results from the Korean Nosocomial Infections Surveillance System (KONIS). J Hosp Infect 2016;92:363-371.

26. Kim B, Kim J, Kim SW, Pai H. A survey of antimicrobial stewardship programs in Korea, 2015. J Korean Med Sci 2016;31:1553-1559.

27. Barlam TF, Cosgrove SE, Abbo LM, et al. Implementing an antibiotic stewardship program: guidelines by the Infectious Diseases Society of America and the Society for Healthcare Epidemiology of America. Clin Infect Dis 2016;62:e51-e77. 


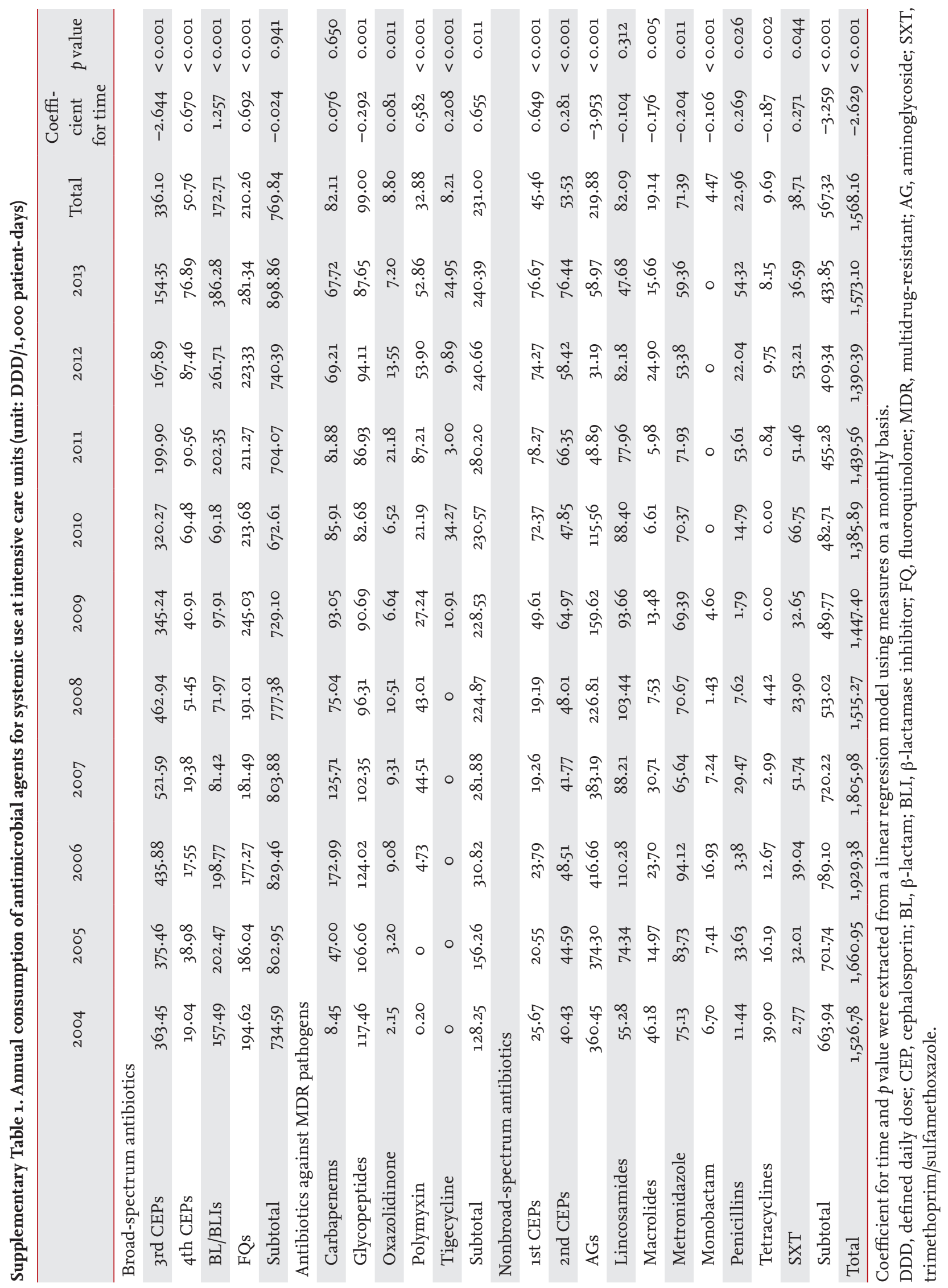




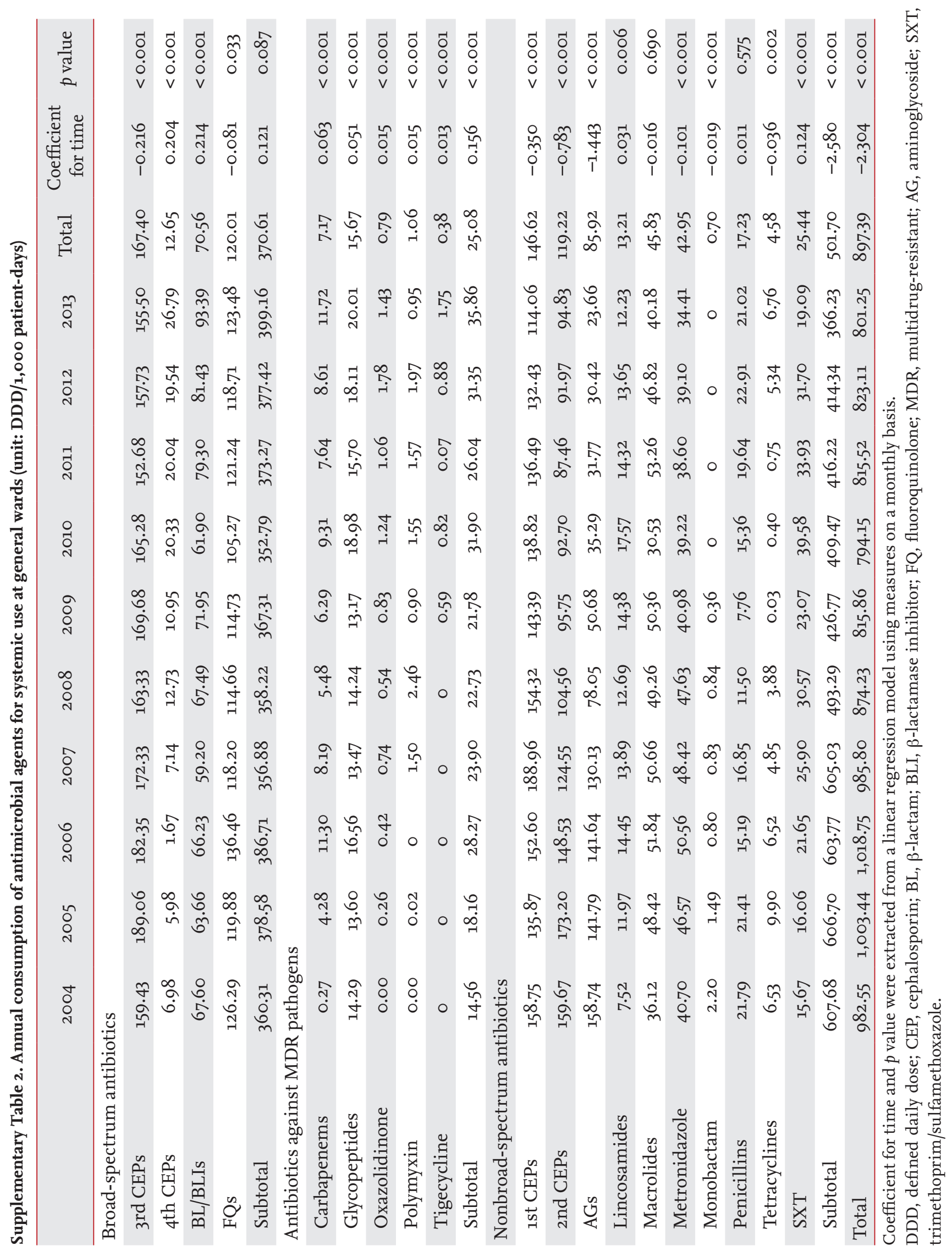




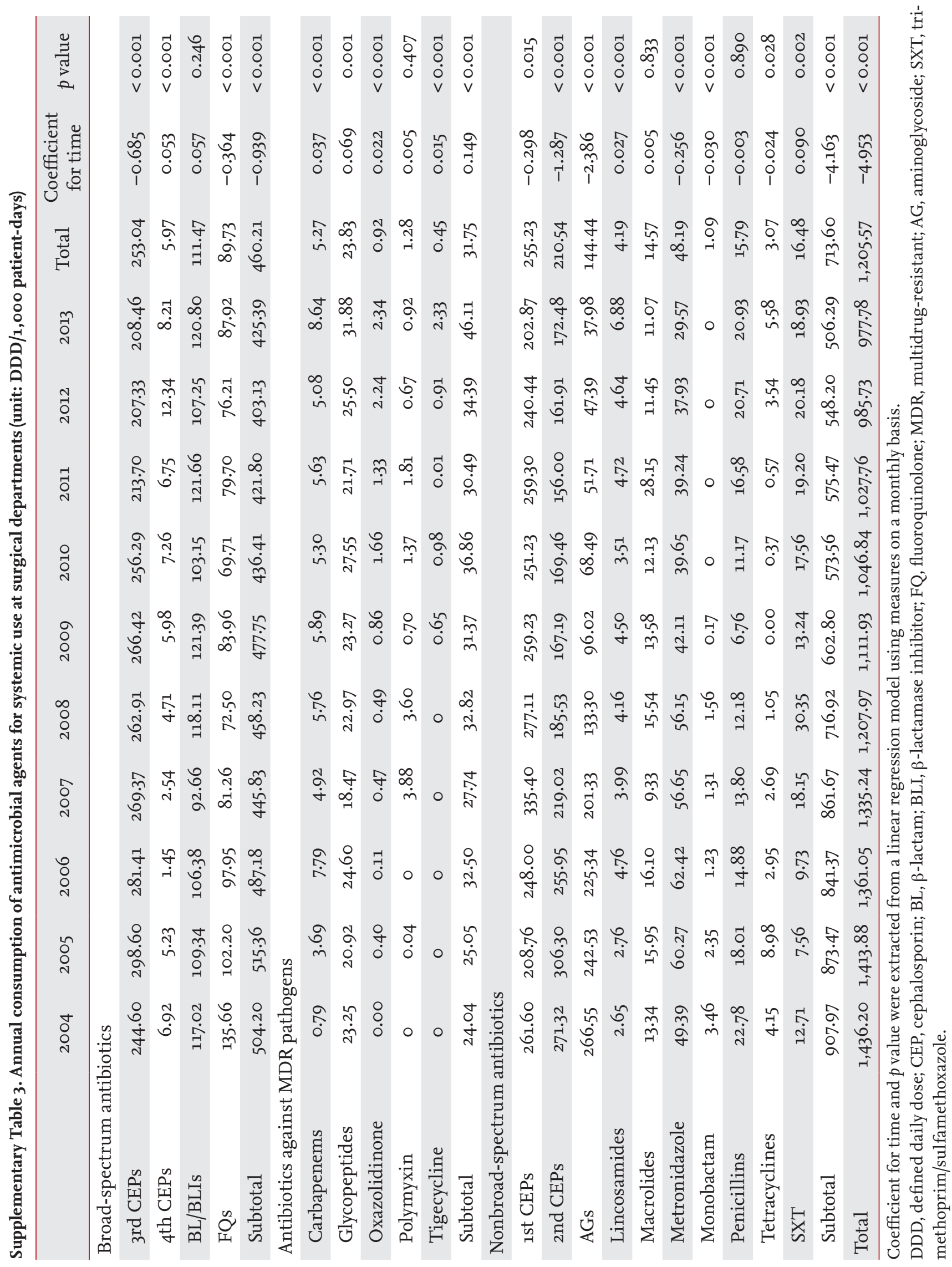




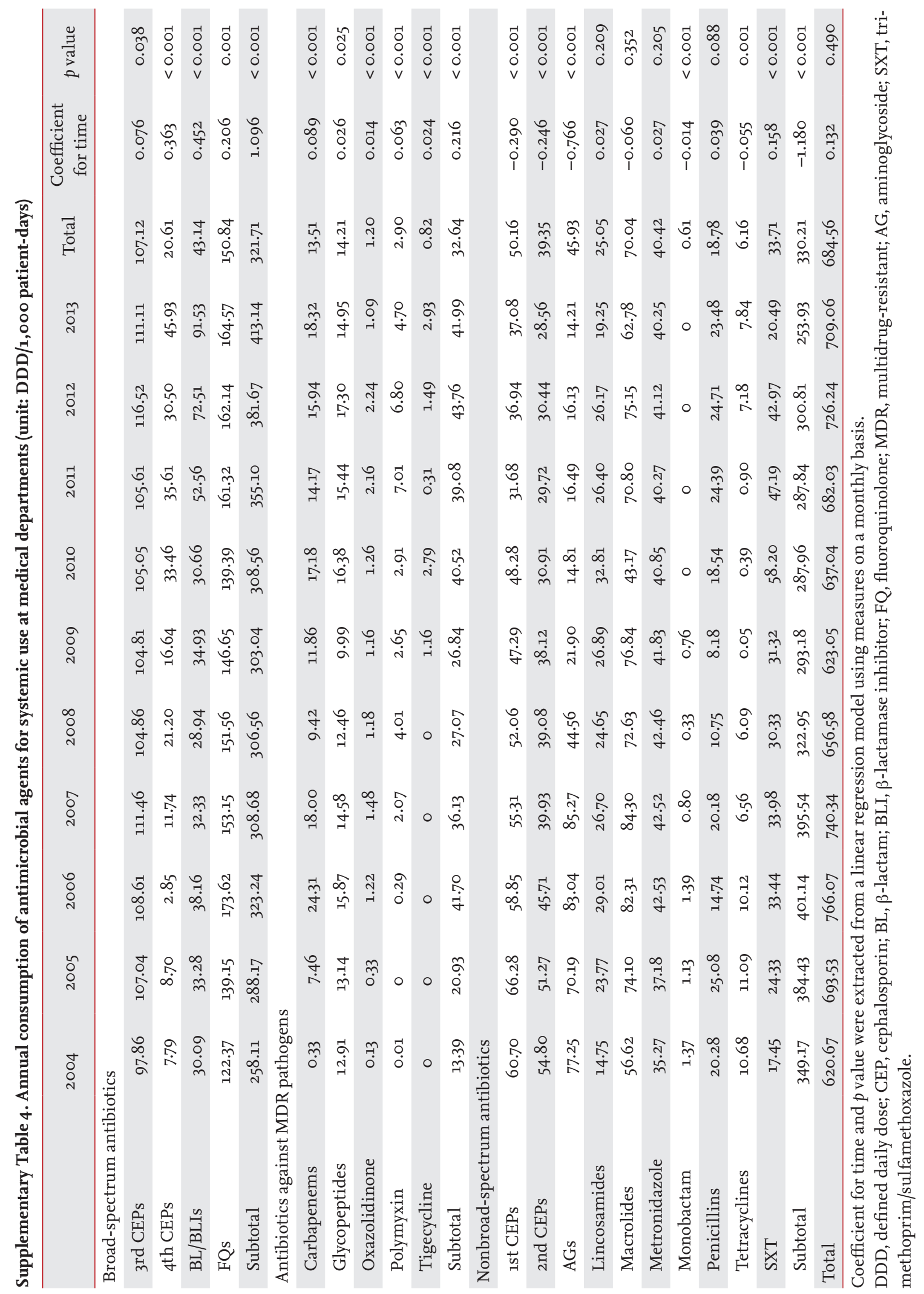

